\title{
Mathematische Spuren in der Philosophie ${ }^{1}$
}

\author{
Knut Radbruch (Kaiserslautern)
}

Seinen Versuch, den Begriff der negativen Größen in die Weltweisheit einzuführen beginnt der neununddreißigjährige Immanuel Kant mit einer grundsätzlichen Erörterung über einen etwaigen „Gebrauch, den man in der Weltweisheit von der Mathematik machen kann ${ }^{62}$. Dabei stellt er die These auf, daß Mathematik grundsätzlich nur auf zweierlei Art in die Philosophie eingreifen könne. Eine erste Möglichkeit sieht Kant in der Nachahmung mathematischer Methoden bei der Darstellung von Philosophie, die andere Möglichkeit besteht für ihn in der konkreten Anwendung mathematischer Theorien in der Naturlehre. Die zuerst genannte Möglichkeit beurteilt Kant ausgesprochen negativ; seine Kritik an dem von Comenius zunächst ganz allgemein formulierten und dann von Christian Wolff insbesondere für die Philosophie favorisierten Programm einer Präsentation der Philosophie nach mathematischem Vorbild - einer Darstellung more geometrico demonstrata - ist hinlänglich bekannt. Die Verwendung von Mathematik in der Naturlehre sieht Kant zwar durchaus positiv; in den Metaphysischen Anfangsgründen der Naturwissenschaft wird er gut zwei Jahrzehnte später sogar jene berühmte Behauptung hinzufügen, ,daß in jeder besonderen Naturlehre nur so viel eigentliche Wissenschaft angetroffen werden könne, als darin Mathematik anzutreffen ist ${ }^{* 3}$. Dennoch weist Kant mit aller Deutlichkeit auf die engen Grenzen des Wirkungsbereichs solcher Anwendungen von Mathematik hin, denn seiner Meinung nach würden ,,aber auch nur die zur Naturlehre

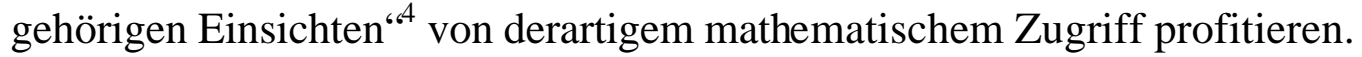

Die Zwischenbilanz ist ernüchternd, im Hinblick auf den Titel dieses Vortrags fast schon deprimierend: Die mathematische Methode ist für die Philosophie ungeeignet, dem Einsatz mathematischer Theorien in der Philosophie sind enge Grenzen gesetzt; andersartige mögliche mathematische Spuren in der Philosophie gibt es für Kant in dieser Arbeit aus dem Jahr 1763 nicht.

Glücklicherweise stellt schon Kant selbst ein ausgesprochen ergiebiges Gegenbeispiel zu seiner eigenen These dar, denn sowohl in seinen vorkritischen als auch in seinen kritischen Schriften finden sich zahlreiche mathematische Spuren. Und Kant ist in dieser Hinsicht keine Ausnahme unter den Philosophen. Bei fast allen abendländischen Philosophen, die originelle neue Philosophie erarbeitet haben, ist die folgende $\mathrm{Ge}$ meinsamkeit nicht zu übersehen: Explizit oder implizit spielt Mathematik eine wesentliche Rolle für die neue philosophische Konzeption; die inhaltliche Auseinandersetzung mit Mathematik prägt den philosophischen Entwurf.

\footnotetext{
${ }^{1}$ Erweiterte Fassung eines Vortrags, der am 20.1.1998 auf Einladung des philosophischen Instituts an der Universität Erlangen gehalten wurde

${ }^{2}$ Kant: Werke 2, S.779

${ }^{3}$ Kant: Werke 8, S.14

${ }^{4}$ Kant: Werke 2, S.779
} 
Die überlieferten philosophischen Texte enthalten somit ein höchst komplexes Mosaik mathematischer Spuren. Seit längerer Zeit schon bemühe ich mich um systematische Spurensuche, Spurensicherung und Spurendeutung eben dieser mathematischen Spuren in der Philosophie. Das erkenntnisleitende Interesse einer derartigen Untersuchung erwuchs aus der Beobachtung, daß keine andere Disziplin in den vergangenen zweieinhalb Jahrtausenden auf Philosophen so herausfordernd gewirkt hat, keine andere Wissenschaft der Philosophie so nachhaltige Impulse vermittelt hat, wie die Mathematik. Fast alle abendländischen Philosophen von Rang beziehen sich in ihrem Werk mehr oder weniger ausführlich auf Mathematik. Derartige Zugriffe seitens der Philosophie auf Mathematik zielen in keinem Fall auf einen Beitrag zur mathematischen Forschung.

Welche Gründe sind es also, die der Mathematik einen so hohen Stellenwert im Denken der großen Philosophen sichern? Mit welchen Intentionen und Zielsetzungen montieren die maßgebenden Philosophen von Platon bis Heidegger, von Aristoteles bis Bloch immer wieder Aussagen zur Mathematik und Reflexionen über Mathematik in ihre Philosophie? Weshalb war in den vergangenen zweieinhalb Jahrtausenden keine andere Wissenschaft für die Philosophie so »frag-würdig « wie die Mathematik? Diesen aus der Sicht der Philosophie gestellten Fragen stehen entsprechende Fragen aus der Mathematik heraus zur Seite: Welche Mathematik wird von der Philosophie assimiliert? Werden von den je einzelnen Philosophen Bausteine der Mathematik quasi als Fertigprodukt einfach übernommen oder erfährt die Mathematik vorab eine spezielle Aufbereitung mit philosophischer Drift? Von einer Analyse all dieser Fragen dürfen sowohl die Philosophie als auch die Mathematik einen Zugewinn an Einsicht in den eigenen wissenschaftstheoretischen und wissenschaftsphilosophischen Status erhoffen - und zwar durch das Wechselspiel von disziplinärer Selbstbeobachtung und Fremdbeobachtung. Dem zur Zeit gehörig überstrapazierten Begriff der Interdisziplinarität liegt ja eine äußerst elementare Frkenntnis zugrunde, daß nämlich zwischen kategorialem Eigenverstehen und kategorialem Fremdverstehen eine unlösbare Wahlverwandtschaft besteht.

Ein derartiges methodisches Vorgehen ist übrigens keineswegs neu, sondern reicht insbesondere im Hinblick auf den Begründungszusammenhang von Mathematik und Philosophie zumindest bis in die Epoche der Aufklärung zurück. 1726 veröffentlichte Christian Wolff eine Ausführliche Nachricht von seinen eigenen Schriften, die er in deutscher Sprache herausgegeben. Darin vertritt er die These, daß „die Disciplinen sich aufeinander beziehen müssen“ und folgert daraus: „Wenn man zu gründlicher Erkäntnis gelangen will, so muß man die Wahrheiten einer Disciplin durch die Wahrheiten der andern verstehen und begreiffen lernen. ${ }^{.5}$ An diese allgemeine Direktive schließt er dann das persönliche Bekenntnis an: „Und eben dieses ist die Ursache, warum ich mich nicht die Mühe verdriessen lassen alle mathematische und philosophische Disciplinen in eine beständige Verknüpffung mit einander zu bringen. “6

Aus dem umfangreichen Quellenmaterial mathematischer Spuren in der Philosophie kann ich in diesem Vortrag nur eine kleine Auswahl präsentieren und thematisieren:

1. Mathematik als Paradigma der Philosophie: Platon

2. Mathematik als Gleichnis der Philosophie: Nikolaus von Kues

\footnotetext{
${ }^{5}$ Wolff: Ausführliche Nachricht, S.666

${ }^{6}$ Ebd., S.667
} 
3. Mathematik als Kontrast der Philosophie: Kant

4. Mathematik als Folie der Philosophie: Schelling

5. Mathematik als Ziel der Philosophie: Friedrich Schlegel

6. Mathematik als Problem der Philosophie: Hegel

7. Mathematik als Exempel der Philosophie: Schopenhauer

8. Mathematik als Klient der Philosophie: Heidegger

Bereits diese Übersicht zeigt signifikant, daß und wie Mathematik auf recht unterschiedliche Art und Weise in die Philosophie hineinwirkt. Die detaillierte Betrachtung der genannten acht Philosophen wird diese Beobachtung voll und ganz bestätigen.

\section{Mathematik als Paradigma der Philosophie: Platon}

„Ich bin überzeugt, daß die griechische Philosophie, dieses in allen Weltkulturen einzigartige Kunstwerk, ohne das mathematische Paradigma undenkbar gewesen wäre. ${ }^{67}$ Diese von C.F. v.Weizsäcker in einer Notiz zu Platons Philosophie des Abstiegs geäußerte Überzeugung ist heute zumindest mehrheitsfähig. Insbesondere Platons Ideenlehre ist ganz offensichtlich mit Blick auf Mathematik konzipiert worden. Mittelstraß spricht explizit von den ,geometrischen Wurzeln der Platonischen Ideenlehre ${ }^{68}$ und Patzig meint, ,daß Platon am Beispiel der Mathematik etwas aufgegangen ist, das er in der Ideenlehre festhielt ${ }^{\triangleleft}{ }^{9}$. Inwiefern fungiert hier Mathematik als Paradigma der Philosophie?

Wir wissen heute, daß es eine bemerkenswert ausgereifte und höchst leistungsfähige vorgriechische Mathematik gegeben hat. Dort wird ein Balken gegebener Länge schräg an eine Hauswand gestellt und es wird verraten, wie hoch die Spitze des Balkens an der Wand postiert ist; daraufhin wird errechnet, wie weit der Fußpunkt des Balkens von der Wand entfernt ist. Die Berechnung erfolgt genau so, wie wir sie heute ausführen würden, nämlich durch Rückgriff auf den Satz des Pythagoras. Allerdings stand der Balken an der Wand tausend Jahre bevor Pythagoras geboren wurde. In einer anderen Aufgabe aus diesen frühen Zeiten ist der Durchmesser eines runden Getreidefeldes gegeben und es wird dann der Flächeninhalt des Feldes bestimmt. Diese Berechnung basiert auf einem verteufelt guten Näherungswert für die Kreiszahl $\pi$, nämlich 3,14. Dabei ist keine Rede von Behauptung und Beweis In dieser babylonischen und ägyptischen Mathematik werden auf konkrete Gegenstände bezogene, praxisorientierte Aufgaben zusammen mit der Lösung in Gestalt einer detaillierten Rechenvorschrift präsentiert. Einziges Wahrheitskriterium ist - unausgesprochen - die pragmatische Bewährung.

Aus ganz anderem Gestein sind die Theoreme des Thales gehauen, die am Anfang der griechischen Geometrie stehen:

In jedem gleichschenkligen Dreieck sind die Basiswinkel gleich.

Der Kreis wird von jedem Durchmesser halbiert.

\footnotetext{
${ }^{7}$ v.Weizsäcker: Zeit und Wissen, S.1085

${ }^{8}$ Mittelstraß: Die geometrischen Wurzeln der Platonischen Ideenlehre

${ }^{9}$ Patzig: Tatsachen, Normen, Sätze, S.126
} 
Hier geht es nicht um Balken, Felder oder Wagenräder, sondern um Dreiecke und Kreise. Und es wird auch nicht der Inhalt eines runden Feldes mit ganz bestimmtem Durchmesser konkret ausgerechnet, sondern hier werden Aussagen mit dem Anspruch von Allgemeingültigkeit formuliert, d.h. es werden Behauptungen aufgestellt. Und diese neuartigen, universellen Aussagen erhalten überdies den Status begründeten Wissens, denn es werden Beweise geführt. Dieser Paradigmenwechsel von vorgriechischer zu griechischer Geometrie bringt eine völlig neue Form des Wissens unter die Menschen. Und genau diesem neuartigen Wissen widmet Platon seine Aufmerksamkeit, indem er zunächst einmal nachfragt, von welchen Objekten diese thaletische Geometrie überhaupt handelt. Und er selbst ist es, der eine originelle Antwort auf die gestellte Frage liefert. Die Sätze der thaletischen Geometrie, so sagt er, gelten für die Idee des Kreises, für die Idee des Dreiecks und zwar bezüglich der Idee der Gleichheit. Sein genialer Einfall besteht also darin, daß er mit den mathematischen Ideen einen Bereich intelligiblen, insbesondere nicht-empirischen Seins postuliert und auf diese Weise die theoretischen Gegenstände nachreicht, für welche die zuvor bereitgestellten theoretischen Sätze des Thales gelten. Diese Platonische Deutung ist raffiniert, aber sie ist -zumindest aus heutiger Sicht - nicht zwingend. Denn sie beschreibt mathematische Sachverhalte durch ein System, welches in dieser Welt gerade nicht vorzufinden ist; es handelt sich um ein theoretisches Erklärungsmodell. Und darin ist von Begriffen und Objekten die Rede, deren einzige Legitimation in ihrer Bewährung innerhalb des Modells besteht. Die Idee des Kreises und die Idee des Dreiecks sind Begriffe, denen ausschließlich im Rahmen dieses Erklärungsmodells Gehalt zukommt. Platons Deutung ist also nicht ohne Alternative; mit Platon beginnt eine bis in die Gegenwart dynamisch fortgeschriebene philosophische Karriere der Mathematik. Und gleich zu Beginn dieser Karriere gehen von ihr Impulse aus für die Entfaltung von Philosophie. Denn die Überzeugungskraft seiner geometrischen Ideenwelt hat Platon offensichtlich ermutigt, nun in umfassenderem Rahmen eine neue Deutung der Beziehung zwischen empirischer Wahrnehmung und theoretischer Aussage zu entfalten. Bei der Frage etwa nach dem Schönen, der Tapferkeit, dem Guten sieht er eine zur Geometrie analoge Situation: In der sensiblen Welt gibt es jeweils nur ungenaue, unvollkommene Realisierungen von Schönheit; die vollkommene Schönheit oder auch Schönheit selbst gibt es in der sensiblen Welt nicht. So postuliert Platon auch hier die Idee der Schönheit, die mit den unvollkommenen Realisierungen von Schönheit durch ein Urbild-Abbild-Verhältnis verknüpft ist. Die Ursprünge von Platons Ideenlehre liegen ohne Zweifel in seiner Interpretation der thaletischen Geometrie.

Diese Rolle der Mathematik für die Entwicklung der Philosophie hat Platon sogar noch systematisch auszunutzen und auszubauen versucht. Weil die Mathematik am Beispiel der Ideen ihre Bewährungsprobe als Paradigma für Philosophie so glänzend bestanden hatte, mußte Platon an einem Ausbau der Mathematik selbst interessiert sein, getragen von der Hoffnung auf neue Impulse für die Philosophie. Und in der Tat berichtet Dikaiarch über die Platonische Akademie: „Es war aber auch ein großer Fortschritt der mathematischen Wissenschaften in jener Zeit zu erkennen, wobei Platon die baumeisterliche Leitung hatte und die Aufgaben stellte, die dann die Mathematiker mit Eifer erforschten. ${ }^{، 10}$ Platon war also nicht nur Architekt seiner Philosophie, er hatte überdies

\footnotetext{
${ }^{10}$ Gaiser: Platons Zusammenschau, S.119
} 
die baumeisterliche Leitung der damaligen Mathematik. Diese beiden Aktivitäten stehen in einem engen Begründungszusammenhang: Platons Philosophie ist auf das Paradigma der Mathematik angewiesen und die Mathematik gewinnt ihren wissenschaftstheoretischen Ort nur innerhalb seiner Philosophie.

\section{Mathematik als Gleichnis der Philosophie: Nikolaus von Kues}

Zu Recht gilt Nikolaus von Kues als derjenige Denker, welcher in der abendländischen Kultur- und Geistesgeschichte dem Übergang vom Mittelalter zur Neuzeit die entscheidenden Impulse vermittelt hat. Dabei entwirft er einen höchst originellen neuartigen Begründungszusammenhang zwischen Theologie, Philosophie und Mathematik. Zunächst nimmt er eine Dreiteilung alles Seienden vor und unterteilt in Sinnliches (reale Welt), Un-Sinnliches (Welt des Geistes) und Über-Sinnliches (Gott). Für ihn hat Gott den Bereich des Sinnlichen nach mathematischen Prinzipien geschaffen: „Gott hat bei der Erschaffung der Welt sich der Arithmetik, der Geometrie, der Musik und der Astronomie bedient..."11 Diesen Schöpfungsprozeß kann der Mensch weder vollends durchschauen noch werkgetreu nachvollziehen. Aber der Mensch simuliert diesen Schöpfungsprozeß in den Wissenschaften. Diese gehören in der geschilderten Dreiteilung zum Bereich des Unsinnlichen, nehmen also eine mittlere Position und damit eine vermittelnde Funktion zwischen der realen Welt und Gott ein. Bei dieser Simulation entspricht dem göttlichen Schöpfungsprozeß ein menschlicher Konstruktionsprozeß. Überhaupt spielt der Begriff der Konstruktion im Wissenschaftsverständnis des Kusaners eine zentrale Rolle. Dies gilt auch und insbesondere für die Mathematik, wo er eine klare Gegenposition zu Platon einnimmt.

Für Platon wird Mathematik ent-deckt bzw. wahr-genommen und insbesondere nicht erfunden oder erschaffen. Damit dieses Entdecken mathematischer Einsicht und Erkenntnis überhaupt stattfinden kann, muß das entsprechende Wissen schon latent in der menschlichen Vernunft angelegt sein. Konsequenterweise ist für Platon die Wiedererinnerung das entscheidende Instrumentarium für Erkenntnisgewinn. Im Phaidon präzisiert Platon die Intention seiner Anamnesislehre knapp und griffig: ,... das Lernen ist eine Erinnerung. " ${ }^{62}$ Für Nikolaus von Kues wird Mathematik nun gerade umgekehrt nicht entdeckt, sondern konstruktiv erschaffen; man kann den Kusaner ohne Übertreibung als Vorläufer oder Urheber des mathematischen Konstruktivismus bezeichnen. Bei ihm ist es ,... unser Geist, der die mathematischen Dinge herstellt ...“" ${ }^{\text {13 }}$ und es ist „die Zahl, die als idealer Gegenstand durch unsere vergleichende Unterscheidung erzeugt wird. "14 Auf diesem Weg gelangt der Mensch zu verläßlichem Wissen. Nun steht aber für den Kusaner jedes Wissen uneingeschränkt im Dienst des Glaubens. Zunächst einmal lassen sich bei ihm philosophische und theologische Argumentation nicht voneinander trennen; und die Wissenschaften - insbesondere die Mathematik - leisten Zubringerdienste für Philosophie und Theologie: ,Wenn wir also die Sache recht betrachtet haben, so besitzen wir nichts Sicheres in unserem Wissen als unsere Mathematik, und diese ist ein Rätselbild,

\footnotetext{
${ }^{11}$ N. v. Kues: Die belehrte Unwissenheit II, S.109

${ }^{12}$ Platon: Phaidon 76a

${ }^{13}$ N. v. Kues: Über den Beryll, S.67

${ }^{14}$ N. v. Kues: Die belehrte Unwissenheit I, S.23
} 
die Werke Gottes zu erjagen. Daher haben bedeutende Männer, wenn sie irgend etwas Großes ausgesprochen haben, es in mathematischem Gleichnis begründet: wie dies, daß die Arten sich zueinander verhalten wie die Zahlen und das Sinnenhafte im Verstandesmäßigen sei wie das Dreieck im Viereck und vieles dergleichen. “15 Wenn hier ,etwas Großes" ausgesprochen wird, so ist damit natürlich stets Einsicht oder Spekulation im Hinblick auf Konzeption und Realisierung des göttlichen Schöpfungsprozesses gemeint. Und die Metapher vom mathematischen Gleichnis als idealem Medium menschlicher Verständigung über göttliches Wirken gehört zu den Lieblingsmetaphern des Kusaners: „Alle unsere weisen und gotterleuchteten Lehrer stimmen darin überein, daß die sichtbaren Dinge in Wahrheit Bilder der unsichtbaren Dinge sind, und daß der Schöpfer auf diese Weise wie im Spiegel und Gleichnis für die Geschöpfe dem erkennenden Blick algänglich wird. “16 Diese Präsentationsform durch ein Gleichnis wird für Nikolaus von Kues darüberhinaus mit mathematischem Gehalt versehen, weil für ihn ,,mathematische Einsichten zum beinahe absolut Göttlichen und Ewigen führen“"17 . Gemäß dieser erkenntnisleitenden Richtlinien wird dann beispielsweise die Trinitätslehre am Begriff des Dreiecks festgemacht. Ganz analog dient die Symmetrie der Kugel als Veranschaulichung für die Harmonie Gottes: „Und wie durch unendliche Kreisbewegungen die Kugel entsteht, so ist Gott für alle Kreisbewegungen - gleichsam als die größte Kugel - das absolut einfache Maß. “18 In den Mutmaßungen erläutert der Kusaner am Modell des Tetraeders Binnenstruktur und wechselseitige Abhängigkeiten von sinnlicher Wahrnehmung (entspricht den Seiten des Tetraeders), Vernunft (wird durch die Kanten repräsentiert) und Verstand (symbolisiert durch die Ecken). In diesem Zusammenhang wird sehr subtil begründet, daß die Vernunft aus sechs Komponenten besteht, während sinnliche Wahrnehmung und Verstand jeweils aus vier Grundbausteinen zusammengesetzt sind.

Das Werk des Nikolaus von Kues enthält eine Vielzahl von Beispielen der geschilderten Art, daß also mathematische Begriffe, Proportionen, Konfigurationen usw. als Modell, Spiegel oder Gleichnis für philosophisch-theologische Argumentationen verwendet werden. Wie schon bei Platon, so beobachten wir auch hier, daß und wie darüberhinaus Philosophie ihrerseits in die Entfaltung der Mathematik hineinwirkt: „Mein Streben geht dahin, aus der Koinzidenz der Gegensätze die Vollendung der Mathematik zu gewinnen." ${ }^{\text {19 }}$ Offensichtlich haben religiöse Motive den Kusaner ermutigt, sich um eine »Vollendung « der Mathematik zu bemühen. Nachdem sich die von ihm vorgefundene Mathematik als Gleichnis oder Spiegel seiner philosophisch-theologischen Weltsicht in überzeugender und vielfältiger Weise bewährt hat, muß zwischen Gottes Schöpfung der Welt und menschlicher Schöpfung der Mathematik ein Prinzip der Passung walten. Da Gottes Schöpfung vollkommen ist, wird sich folgerichtig auch die Mathematik zu einer Vollkommenheit vorantreiben und also vollenden lassen. Der mathematische Ertrag dieser Vollendungsbemühungen des Kusaners ist nicht der Rede wert, sie legen aber ein weiteres Zeugnis ab von seiner Grundüberzeugung einer Ausnahmestellung der Mathe-

\footnotetext{
${ }^{15}$ N. v. Kues: Dreiergespräch über das Können-Ist, S.53

${ }^{16}$ N. v. Kues: Die belehrte Unwissenheit I, S.41

${ }^{17}$ N. v. Kues: Die mathematischen Schriften, S.160

${ }^{18}$ N. v. Kues: Die belehrte Unwissenheit I, S.95

${ }^{19}$ N. v. Kues: Die mathematischen Schriften, S.161
} 
matik unter den Wissenschaften im Rahmen religiöser Weltorientierung und menschlicher Interpretation der göttlichen Schöpfung.

\section{Mathematik als Kontrast der Philosophie: Kant}

Mathematische Spuren durchziehen Kants Werk in allen Schaffensperioden. Ob sich der Zweiundzwanzigjährige Gedanken von der wahren Schätzung der lebendigen Kräfte macht, ob sich der Vierzigjährige vergeblich bemüht, mit einer Untersuchung über die Deutlichkeit der natürlichen Theologie und der Moral den Preis der Königlichen Akademie der Wissenschaften in Berlin zu erringen, oder ob der vierundsiebzigjährige eine Anthropologie in pragmatischer Hinsicht verfaßt - überall finden sich Einschübe zur Mathematik, immer wieder verleihen Anmerkungen zur Mathematik den auBermathematischen Argumentationen Antrieb und Überzeugungskraft.

Will man die Art und Weise des Kantschen Zugriffs auf Mathematik knapp und prägnant begrifflich fassen, so bietet sich als Formulierung an, daß Kant durchgängig Tragweite und Grenzen der Mathematik analysiert. Im Zusammenhang mit Kants philosophischen Bemühungen fungiert die Mathematik dabei bevorzugt als Kontrast zur Philosophie. Das sei kurz skizziert.

In der Vorrede zur zweiten Auflage der Kritik der reinen Vernunft präzisiert Kant den Unterschied zwischen Wissenschaft und Herumtappen: „Ob die Bearbeitung der Erkenntnisse, die zum Vernunftgeschäfte gehören, den sicheren Gang einer Wissenschaft gehe oder nicht, das läßt sich bald aus dem Erfolg beurteilen ... wenn es nicht möglich ist, die verschiedenen Mitarbeiter in der Art, wie die gemeinschaftliche Absicht erfolgt werden soll, einhellig zu machen: so kann man immer überzeugt sein, daß ein solches Studium bei weitem noch nicht den sichern Gang einer Wissenschaft eingeschlagen, sondern ein bloßes Herumtappen sei ... ${ }^{20}$ Den Status einer Wissenschaft billigte Kant nur der Mathematik und der mathematischen Naturwissenschaft zu. „Die Mathematik ist von den frühesten Zeiten her, wohin die Geschichte der menschlichen Vernunft reicht, in dem bewundernswürdigen Volke der Griechen den sichern Weg einer Wissenschaft gegangen. ${ }^{621}$ Die Philosophie hingegen, und hier insbesondere die Metaphysik, befand sich seiner Meinung nach noch in der Mitte des 18. Jahrhunderts im Stadium des Herumtappens. Das Ziel seiner kritischen Philosophie sah er in der Beförderung von Philosophie aus der niederen Stufe des Herumtappens in den höheren Rang einer Wissenschaft. Auf dem Weg zu diesem Ziel geht er mit bewundernswerter methodischer Raffinesse vor. Er entwirft einerseits eine völlig neuartige Konzeption für den wissenschaftstheoretischen Status von Mathematik und arbeitet andererseits detailliert die grundlegende wissenschaftstheoretische Differenz zwischen Mathematik und Philosophie heraus. Aus der gesicherten Wissenschaft Mathematik und der methodisch bestimmten Differenz zwischen Mathematik und Philosophie hofft er auch Philosophie als Wissenschaft einzufangen.

Die neue Auffassung von Mathematik brauche ich nur in Erinnerung zu rufen. Kant hat im weiteren Umfeld der Mathematik einen Vielfrontenkrieg geführt, zwar nicht direkt gegen die Wissenschaft Mathematik, aber sowohl gegen überkommene Auffassungen von

\footnotetext{
${ }^{20} \mathrm{KdrV}, \mathrm{B}$ VII

${ }^{21} \mathrm{KdrV}, \mathrm{BX}$
} 
Mathematik als auch gegen gängige Gewohnheiten des Umgangs mit Mathematik. Was die Auffassung von Mathematik betrifft, bezieht er eine doppelte Opposition, nämlich sowohl gegen die englischen Empiristen als auch gegen den von Leibniz vertretenen analytischen Charakter der Mathematik. Im Hinblick auf den Umgang mit Mathematik rügt er insbesondere die Verwendung der mathematischen Methode in der Philosophie.

Mathematische Erkenntnis ist für Kant sowohl synthetisch - hier bezieht er Opposition gegen Leibniz - als auch a priori - hier nimmt er eine Gegenposition zu den englischen Empiristen ein. Die Einsicht, daß mathematische Erkenntnis synthetisch und a priori ist, sieht Kant im Ursprung zuerst in der thaletischen Geometrie realisiert. Sowohl Platon als auch Kant nehmen Bezug auf die thaletische Geometrie, jedoch jeweils in einer Art und Weise, wie sie unterschiedlicher kaum denkbar ist. Platon fragt angesichts der thaletischen Geometrie nach den Gegenständen der Erkenntnis, Kant fragt gerade gegenläufig dazu nach der Erkenntnis der Gegenstände. Mit Hinweis auf die historischen Ursprünge bei den Griechen stellt Kant also fest: Mathematische Erkenntnis ist synthetisch und a priori. Dem stellt er die programmatische Aussage zur Seite: Metaphysische Erkenntnis muß - will sie den Rang einer Wissenschaft beanspruchen - ebenfalls synthetisch und a priori sein.

Diesem Gleichklang im Typus von mathematischer und metaphysischer Erkenntnis synthetisch und a priori - stellt Kant nun prägnante Differenzen zur Seite, die Mathematik und Philosophie in ein kontradiktorisches Verhältnis zueinander setzen. So weist er darauf hin, daß Definitionen, Demonstrationen und Axiome für die Mathematik konstitutiv sind, nicht jedoch für die Philosophie. In der Transzendentalen Methodenlehre findet sich der vielzitierte Satz: „Die philosophische Erkenntnis ist die Vernunfterkenntnis aus Begriffen, die mathematische aus der Konstruktion der Begriffe. ${ }^{622}$ Daraus wird dann gefolgert: „Die philosophische Erkenntnis betrachtet also das Besondere nur im Allgemeinen, die mathematische das Allgemeine im Besonderen... ${ }^{\text {223 }}$ Über den Gleichklang im Charakter der Erkenntnis von Mathematik und Metaphysik, dessen Realisierung in der Mathematik, sowie den Methodenkontrast der Betrachtungsweise in Mathematik und Philosophie bestimmt Kant also ,,jede künftige Metaphysik, die als Wissenschaft wird auftreten können“.

Kaum ein Philosoph hat die nachfolgenden Mathematikergenerationen so herausgefordert wie Kant. Die Urteile der Mathematiker loten das breite Spektrum von euphorischer Zustimmung bis zu schroffer Ablehnung voll aus

\section{Mathematik als Folie der Philosophie: Schelling}

Es sei zunächst an Kants Gegenüberstellung von Mathematik und Philosophie erinnert, die er 1781 in der 1. Auflage seiner Kritik der reinen Vernunft unüberbietbar griffig formuliert: „Die philosophische Erkenntnis betrachtet also das Besondere nur im Allgemeinen, die mathematische das Allgemeine im Besonderen ... ${ }^{24}$ Genau zweiundzwanzig Jahre später heißt es bei Schelling in seinen Vorlesungen über das akademische Studium: „Philosophie und Mathematik sind sich darin gleich, daß beide in der absoluten

\footnotetext{
${ }^{22} \mathrm{KdrV}, \mathrm{B} 741$

${ }^{23} \mathrm{KdrV}, \mathrm{B} 742$

${ }^{24} \mathrm{KdrV}, \mathrm{B} 742$
} 
Identität des Allgemeinen und Besonderen gegründet ... sind ‘25. Man könnte hier ohne Übertreibung von einer zweiten Kopernikanischen Wende der Denkart sprechen.

Die absolute Identität von Besonderem und Allgemeinem - sowohl in der Mathematik als auch in der Philosophie - sind natürlich als Kernaussage von Schellings Identitätsphilosophie zu lesen. Schellings frühen Texten zur Naturphilosophie läßt sich nun entnehmen, daß er diese absolute Identität durch eine neue Interpretation von Besonderem und Allgemeinem in der Mathematik vorbereitet, welche er dann als Folie seiner philosophischen Konzeption unterlegt.

In der Transzendentalen Methodenlehre demonstriert Kant den Unterschied von Besonderem und Allgemeinem in der Mathematik am Dreieck: „Die einzelne hingezeichnete Figur ist empirisch, und dient gleichwohl, den Begriff, unbeschadet seiner Allgemeinheit, auszudrücken, weil bei dieser empirischen Anschauung ... von Verschiedenheiten, die den Begriff des Triangels nicht verändern, abstrahiert wird. ${ }^{\text {'26 }}$ Zwischen Besonderem und Allgemeinem wird hier sorgfältig unterschieden. Schelling hingegen ebnet den Unterschied radikal ein: „Das Dreieck, welches der Geometer konstruiert, ist für ihn das Urbild selbst, denn es ist für ihn, d.h. für seine eigentümliche Anschauung, absolute Einheit des Allgemeinen und Besonderen .... ${ }^{27}$. Schelling nimmt also nicht nur im Hinblick auf das Verhältnis von Mathematik und Philosophie, sondern auch in bezug auf die Mathematik eine klare Gegenposition zu Kant ein.

In seiner Philosophie der Offenbarung gibt uns Schelling einen Wink, wie man sich einem Philosophen nähern sollte: "Will man einen Philosophen ehren, so muß man ihn da auffassen, wo er noch nicht zu den Folgen fortgegangen ist, in seinem Grundgedanken; denn in der weiteren Entwicklung kann er gegen seine eigene Absicht irren, und nichts ist leichter als in der Philosophie zu irren, wo jeder falsche Schritt von unendlichen Folgen ist, wo man überhaupt auf einem Weg sich befindet, der von allen Seiten von Abgründen umgeben ist. Der w a h r e Gedanke eines Philosophen ist eben sein Grundgedanke, der von dem er ausgeht. ${ }^{128}$ Befolgen wir also diesen Wink und fragen nach Schellings Grundgedanken in bezug auf die Mathematik.

Dazu müssen wir uns zu allererst um ein Verständnis seiner These von der absoluten Einheit des Allgemeinen und Besonderen in der Mathematik bemühen. Wenn mathematische Erkenntnis und mathematischer Gegenstand als Getrennte aufgefaßt bleiben und zugleich die Einheit von Allgemeinem und Besonderem gleichsam von außen, nämlich von der Erkenntnis her, betrachtet oder vollzogen werden soll, ist diese Einheit unverständlich, wenn nicht gar widersprüchlich. Sie gelingt dann und nur dann, wenn ihr eine zweite Einheit vorgelagert wird, nämlich die von mathematischer Erkenntnis und mathematischem Gegenstand, also von mathematischem Denken und mathematischem Sein. Falls Erkenntnis und Gegenstand als untrennbare Einheit zugrunde liegen, hat es nicht nur Sinn, Allgemeines und Besonderes als Einheit aufzufassen, sondern diese zweite Einheit folgt sogar aus der ersten mit systemimmanenter Notwendigkeit. Diese doppelte Einheit von mathematischer Erkenntnis und mathematischem Gegenstand sowie von Allgemeinem und Besonderem, ist nun genau Schellings Grundgedanke im Hinblick auf Mathe-

\footnotetext{
${ }^{25}$ Schelling: Werke I.5; S.255

${ }^{26} \mathrm{KdrV}$, B 741/742

${ }^{27}$ Schelling: Werke I.4, S.347

${ }^{28}$ Ebd. II.3, S.60
} 
matik. Von diesem Grundgedanken ausgehend lassen sich fast alle Anmerkungen Schellings zur Mathematik in konsistenter Weise deuten und einordnen. Bevor dies an einigen typischen Beispielen demonstriert wird, sollen jedoch einige grundsätzliche Überlegungen Schellings zum Einheitsbegriff in den Blick genommen werden. Bei Schelling werden bis dahin getrennte, teilweise konträre Begriffspaare einer Einheit untergeordnet: Besonderes - Allgemeines, Denken - Sein, Subjekt - Objekt, Endlich - Unendlich usw. Die Konstituenten eines Paars verlieren auch in der Einheit nicht ihre Eigenständigkeit; es wird vielmehr in einer der beiden Komponenten die zugehörige Einheit erklärt bzw. dargestellt und dabei hängt die jeweilige Erscheinungsform oder Darstellungsweise der Einheit von der dazu gewählten Komponente ab. So kann die Einheit von Denken und Sein in der Mathematik sowohl im Denken als auch im Sein festgemacht werden - den ersten Weg sieht Schelling in der Arithmetik realisiert, auf dem zweiten Weg erklärt bei ihm die Geometrie die Einheit: "So kann daraus, daß Geometrie und Arithmetik, jede ... diese Einheit, die eine ... im Seyn, die andere ... im Denken ausdrückt, zugleich die Einheit und die Verschiedenheit der geometrischen und arithmetischen Anschauung hinreichend begriffen werden. ${ }^{629}$ Eine andere Ausformung erhält Schellings Einheitskonzeption von Besonderem und Allgemeinem in der Mathematik, wenn er meint, "daß die Arithmetik ein Besonderes (Verhältniß von einzelnen Größen) im Allgemeinen, die Geometrie ein Allgemeines (den Begriff einer Figur) im Besonderen ausdrückt.."30 Einheit und Unterschied bilden ebenfalls ein zusammengehörendes Paar; die Einheit wird auf jeweils charakteristische Weise zugleich mit der Eigenständigkeit der Komponenten gedacht. Besonders deutlich schildert Schelling diese Zusammenhänge im System des transzendentalen Idealismus am Beispiel des Begriffspaars Subjektives - Objektives. Zunächst wird die Einheit klar herausgestellt: "Im Wissen selbst - indem ich weiß - ist Objektives und Subjektives so vereinigt, daß man nicht sagen kann, welchem von beiden die Priorität zukomme. Es ist hier kein Erstes und kein Zweites, beide sind gleichzeitig und Eins. ${ }^{31}$ Es folgt dann sofort der Hinweis, daß man für eine Erläuterung dieser Einheit notwendig mit einem der beiden Begriffe beginnen müsse, jedoch "von welchem von beiden ich ausgehe, ist durch die Aufgabe nicht bestimmt. ${ }^{132}$ Beginnt man mit dem Objektiven, so gelangt man zunächst zur Naturphilosophie; zur Transzendentalphilosophie hingegen führt der mit dem Subjektiven einsetzende Weg. Beide Wege dokumentieren trotz unterschiedlicher Startpositionen und entgegengesetzter Laufrichtung die Einheit von Objektivem und Subjektivem.

Wir hatten auf die Orientierungsfunktion der Mathematik für die Konzeption von Platons Ideenlehre hingewiesen. Analog hat sich Kant im Übergang von seiner vorkritischen zur kritischen Philosophie eng an der Mathematik orientiert. Man darf annehmen, daß auch Schelling am Beispiel der Mathematik Zusammenhänge und Abhängigkeiten erkannte, die er dann für den Entwurf seiner Identitätsphilosophie aufgriff und entfaltete. Von einer solchen Annahme ausgehend könnten sich sogar neue Zusammenhänge in der Philosophie Schellings aufschließen. Am Beispiel der Mathematik wird nämlich deutlich, daß Einheiten bzw. Einheitsoperationen in einem Verbund, und nicht unabhängig

\footnotetext{
${ }^{29}$ Ebd. I.4, S.347

${ }^{30}$ Ebd. I.5, S.130/131

${ }^{31}$ Ebd. I.3, S.339

${ }^{32}$ Ebd. I.3, S.340
} 
voneinander in den Blick kommen. Analog liegt der Identitätsphilosophie von Schelling ganz offensichtlich ein komplexes Mosaik von Einheits-Bausteinen zugrunde, wobei die einzelnen Bauelemente in jeweils mehrfacher Weise sowohl als Resultat wie auch als Anlaß solcher Einheitsoperationen fungieren.

Im Hinblick auf die Philosophie bedarf ein grundlegender Unterschied zwischen Kant und Schelling noch der Erläuterung und Erklärung. Die Tatsache, daß bei Kant die Philosophie das Besondere im Allgemeinen und nicht wie die Mathematik das Allgemeine im Besonderen betrachtet, hat ja seinen Grund darin, daß nur die Mathematik das Mittel der Konstruktion zur Verfügung hat, nicht jedoch die Philosophie. Wenn nun Schelling in scharfem Gegensatz zu Kant sowohl Mathematik als auch Philosophie durch die Identität von Allgemeinem und Besonderem charakterisiert, muß also das Argument mit der Konstruktion hinfällig geworden sein. Und genau dies wird von Schelling in der Weise aufgegriffen, daß er die Konstruktion auch in der Philosophie etabliert: "Die Lehre von der philosophischen Konstruktion wird künftig eines der wichtigsten Kapitel in der wissenschaftlichen Philosophie ausmachen... ${ }^{33}$ Den Gleichklang von Mathematik und Philosophie mit der Identität von Allgemeinem und Besonderem erreicht Schelling durch eine neue Deutung von Konstruktion in der Mathematik und durch jene neue Einführung von Konstruktion in die Philosophie. Was für viele Philosophen vor ihm gilt, trifft auch für Schelling zu: Seine Auffassung von Mathematik und die Philosophie, zu deren Entfaltung diese Auffassung von Mathematik an zentralen Positionen in Gebrauch genommen wird, bilden eine architektonische Einheit. Schellings Philosophie und seine Auffassung von Mathematik erfüllen das Prinzip der Passung.

\section{Mathematik als Ziel der Philosophie: Friedrich Schlegel}

Im Sommer 1798 schrieb Friedrich Schlegel seinem Freund Schleiermacher, daß ihn die Korrespondenz mit Novalis zu einem Studium der Physik zwingen werde: „Mein Briefwechsel mit Hardenberg wird wohl sehr physikalisch werden. Ich muß doch diese Wissenschaft eben auch lernen ... Indessen ist mir doch etwas bange, indem ich mich auf ein so fremdes Feld wage, auf dem ich wohl immer nur Gast seyn werde (mit der Mathematik wäre es etwas anders). ${ }^{634}$ In der Mathematik fühlte sich Schlegel also nicht als Gast. Er war in der Tat mit mathematischen Begriffen und Denkformen sowie mit der mathematischen Formel- und Symbolsprache gut vertraut, obwohl er nie den Anspruch erhoben hat, als Mathematiker anerkannt und angesehen zu werden. Aber mathematische Spuren durchziehen sein gesamtes Werk. Eine dieser Spuren sei lediglich genannt, einer anderen soll sorgfältig nachgegangen werden.

In Schlegels Fragmenten, Vorlesungen und Aufsätzen kehren zwei methodische Prinzipien immer wieder. Da ist einerseits das Bemühen, durch interdisziplinäre Betrachtung und Argumentation Einsichten zu gewinnen, die sich isoliert gar nicht erreichen oder formulieren lassen. Ein typisches Beispiel dafür ist sein Entwurf einer Poetik unter Rückgriff auf Mathematik. Andererseits unternimmt Schlegel immer wieder den Versuch, verschiedene Kulturleistungen nach systematischen Gesichtspunkten zu strukturie-

\footnotetext{
${ }^{33}$ Ebd. I.5, S.125

${ }^{34}$ KA XXIV, S.151/162
} 
ren und damit in einer übergeordneten Einheit zusammenzuführen. Ein besonders aufschlußreiches Exempel für diese Vorgehensweise ist seine Synopse von Wissenschaft und Kunst. Dabei tritt ebenfalls die Mathematik auf den Plan. Diese Spur wollen wir hier verfolgen.

In seiner Vorlesung über europäische Literatur bestimmt Schlegel zunächst die Philosophie ,als allgemeinste Wissenschaft: als Wissenschaft der Wissenschaften“ und die

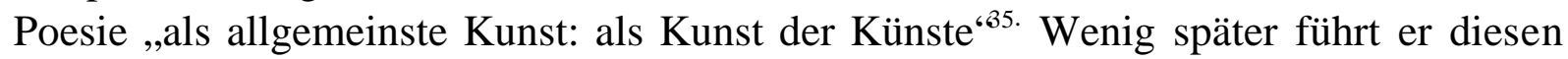
Gedanken noch weiter aus: „Die Poesie vereinigt alle Kunst, die Philosophie ist Wissenschaft aller Wissenschaften. Poesie ist Musik, ist Malerei in Worten; die Philosophie vereinigt den Geist, die Form und Methode aller anderen Wissenschaften ... Philosophie und Poesie sind sozusagen die eigentliche Weltseele aller Wissenschaften und Künste und der gemeinschaftliche Mittelpunkt. ${ }^{636}$ Nun fehlt nur noch die Zusammenführung von Philosophie und Poesie, und von einer derartigen Synopse hat Schlegel klare Vorstellungen: „Eine Wissenschaft, die ... alle Künste und Wissenschaften in eine verbindet, die also die Kunst wäre, das Göttliche zu produziren, könnte mit keinem andern Namen bezeichnet werden als MAGIE. ${ }^{67}$ Man beachte das Wortspiel: „Eine Wissenschaft, die ... also die Kunst wäre“. Diese Disziplin würde also Wissenschaft und Kunst zugleich sein und als solche sämtliche Wissenschaften und Künste in einer Einheit zusammenfassen. Schlegel gibt an dieser Stelle nur den Namen für diese exzeptionelle Disziplin preis: MAGIE. Man muß also Schlegels mehr als 7000 Fragmente daraufhin durchsehen, was mit dieser Magie gemeint sein könnte. Einen ersten Hinweis gibt das folgende Fragment: „Die Principien der Musik und der Magie sind in Mathematik zu suchen. ${ }^{638}$ Wenn in dieser Weise Magie auf Mathematik zurückgeführt wird, muß nun nach den Prinzipien der Mathematik gefragt werden. Und dazu heißt es bei Schlegel: „Die Principien der Mathematik sind magisch. ${ }^{69}$ Es bestimmen sich also Mathematik und Magie wechselseitig; beide sind sowohl Voraussetzung als auch Konsequenz des Partners. Aber Schlegel geht noch einen Schritt weiter, wenn er in einem anderen Fragment notiert: „Mathematik = Magie ... Mathematik wäre dann wohl ein Organon der Magie. ${ }^{\text {(40 }}$ Und die Magie, dies sei in Erinnerung gerufen, trat bei der Suche nach einer Synopse von Philosophie und Poesie und damit auf dem Weg zu einer Einheit aller Wissenschaften und Künste auf den Plan. Zunächst hatte Schlegel nur vorsichtig angedeutet, diese mit universeller Integrationskraft versehene Disziplin „könnte mit keinem anderen Namen bezeichnet werden als MAGIE“. Inzwischen ist klargeworden, was sich für ihn hinter der Magie verbirgt. Es ist dies die Mathematik. Und damit erhält auch ein anderes Fragment, welches isoliert gelesen unverständlich bleibt, eine natürliche Plazierung und Deutung: „Die Mathematik ist die Wissenschaft schlechthin, nicht die mit der man anfängt, sondern die mit der man endigt. ${ }^{\text {‘1 } 1}$ In diesem Sinne fungiert also Mathematik als höchste Wissenschaft und höchste Kunst zugleich, sie läßt alle Wissenschaften und alle Künste als Einheit sichtbar werden.

\footnotetext{
${ }^{35}$ KA XI, S.7

${ }^{36}$ KA XI, S.10

${ }^{37}$ KA XII, S.105

${ }^{38}$ KA XVIII, S.369

${ }^{39}$ KA XVIII, S.154

${ }^{40}$ KA IXX, S.10

${ }^{41}$ KA XVIII, S.137
} 
Zum besseren Verständnis dieser Argumentation sei darauf hingewiesen, daß sich gegen Ende des 18. Jahrhunderts - genauer: in der Frühromantik - ein neuer, insbesondere ausgesprochen positiver Magie-Begriff herausgebildet hat. Und es deutet vieles darauf hin, daß diese damalige neue Auffassung von Magie entlang der Mathematik eingeführt und semantisch fixiert wurde, bevor die Magie als eigenständiger und gefestigter Begriff in die Disziplinenlandschaft entlassen wurde.

Bei Novalis heißt es: „Ächte Mathematik ist das eigentliche Element des Magiers. “42 In einem anderen Fragment stellt Novalis einen Begründungszusammenhang zwischen synthetischen Urteilen a priori und magischer Intelligenz her. ${ }^{43}$ Erst im Anschluß an diese Begriffsklärung und Begriffsfestigung argumentieren Novalis und Schlegel auch in anderen Bereichen mit dem Begriff der Magie. Und dabei geht es in allen Fällen - im Recht, in der Religion, in der Kunst - um höchste bzw. letzte Einsichten oder auch Visionen.

\section{Mathematik als Problem der Philosophie: Hegel}

In seiner Rede, die er 1923 während des Fest-Kolloquiums anläßlich des einhundertsten Geburtstags von Kronecker gehalten hat, betont Adolf Kneser, es sei „für die Wissenschaftsgeschichte von Interesse, bei drei großen Mathematikern, Jacobi, Kummer und Kronecker, die offenbaren Einflüsse der Hegelschen Philosophie auf ihre geistige Entwicklung nachzuweisen, trotz der bekannten tollen Konstruktionen und Kritiken, durch die sich der Philosoph auf mathematischem Gebiet kompromittiert hat ${ }^{\text {*44 }}$. Den Hegelschen Spuren bei den drei genannten Mathematikern soll hier nicht nachgegangen werden, zumal Kummer seinen Plan einer Enzyklopädie der mathematischen Wissenschaften nach dem Vorbild von Hegels Enzyklopädie der philosophischen Wissenschaften im Grundrisse niemals realisiert hat. Den Vorwurf, daß sich Hegel auf mathematischem Gebiet kompromittiert habe, wird sich heute allerdings kein Mathematiker, der Hegel wirklich gelesen hat, mehr leisten können.

Es gibt für Hegel drei Wege zur Erkenntnis: mittels des Begriffs, über die Empirie und per Verstand. Der Begriff ist konstituierend für die Philosophie: ,,... wie überhaupt der ganze Fortgang des Philosophierens ... nichts anderes ist als das bloße Setzen desjenigen, was in einem Begriffe schon enthalten ist. ${ }^{\text {(45 }}$ Hiervon wird die Mathematik scharf abgegrenzt: ,... denn sie [die Mathematik] ist nicht eine Wissenschaft, die es mit den Begriffen ihrer Gegenstände zu tun ... hätte. ${ }^{\star 46}$ Zumindest Geometrie und Arithmetik sind für Hegel Verstandeswissenschaften. Davon wird noch ausführlich die Rede sein. Mit Hilfe der Empirie schließlich gewinnen die Naturwissenschaften ihre Resultate; auch gegen diese Weise der Erkenntnisgewinnung grenzt Hegel die Mathematik mit aller Deutlichkeit ab, wenn er erklärt, daß ,der Versuch, Beweise von Naturverhältnissen eigentlich mathematisch, d.h. weder aus der Empirie noch aus dem Begriffe zu führen, ein widersinniges Unternehmen ist. ${ }^{\text {“47 }}$ Geometrie und Arithmetik sind für Hegel - wie be-

\footnotetext{
${ }^{42}$ Novalis III, S.593

${ }^{43}$ Ebd. S. 419

${ }^{44}$ Jahresbericht der DMV Bd.33 (1925), S.217

${ }^{45}$ HW VIII, S. 188

${ }^{46} \mathrm{HW}$ V, S.280/281

${ }^{47} \mathrm{HW}$ V, S.407
} 
reits gesagt- reine Verstandeswissenschaften: „Die Wissenschaft der Geometrie hat zu finden, welche Bestimmungen folgen, wenn gewisse andere vorausgesetzt sind... Dies ist die Weise, wie die Geometrie, als abstrakte Verstandeswissenschaft, wissenschaftlich verfährt. ${ }^{‘ 8}$, ,... die Figuren der Arithmetik sind wieder der Verstandesbestimmungen nach Gleichheit und Ungleichheit, der Identifizierung und des Unterscheidens fähig.‘99

Damit haben sowohl Geometrie als auch Arithmetik innerhalb einer Klassifikation von Erkenntnisweisen ihren Platz gefunden, nämlich als Erkenntnis durch den Verstand. Diesen wissenschaftstheoretischen Ort weist Hegel der Mathematik schlechthin zu: „Allein da die Mathematik einmal die Wissenschaft der endlichen Größenbestimmungen ist, ..., so ist sie wesentlich eine Wissenschaft des Verstandes. ${ }^{، 50}$ Im Unterschied zu Kant betrachtet Hegel nun aber nicht nur die traditionellen mathematischen Disziplinen Geometrie und Arithmetik, sondern er interessiert sich auch engagiert für die zu seiner Zeit aktuelle Entwicklung der Analysis. Und hier ist Hegel der Meinung, daß sich die Differential- und Integralrechnung in das geschilderte Verstandeskonzept nicht einfügt: „Den Begriff aber kann die Mathematik ... hier nicht umgehen ... Die Operationen, die sie sich als Differential- und Integralrechnung erlaubt, sind daher der Natur bloß endlicher Bestimmungen und deren Beziehungen gänzlich widersprechend und hätten darum ihre Rechtfertigung allein in dem Begriff. “51 Damit wird aber der zuvor abgesteckte Rahmen von Mathematik gesprengt und die Sache gerät zwangsläufig in den Wirkungsbereich der Philosophie. Und in der Tat werden zentrale Fragen der Analysis bei Hegel zu einem Problem der Philosophie: „Andere mathematische Bestimmungen, wie das Unendliche, Verhältnisse desselben, das Unendlichkleine, Faktoren, Potenzen u.s.f. haben ihre wahrhaften Begriffe in der Philosophie selbst. ${ }^{\text {‘52 }}$

$\mathrm{Zu}$ Hegels Zeit wird in der Differential- und Integralrechnung - im Unterschied zur Geometrie und Arithmetik - nicht nur mit endlichen Größen, sondern auch mit unendlich großen und mit unendlich kleinen Größen gearbeitet. Hegel ist in diesem Zusammenhang der Überzeugung, daß das Unendliche der Mathematik nur angemessen mit Hilfe des Begriffs und also mit philosophischen Methoden beherrscht werden kann. Darüberhinaus läßt sich für ihn das Unendliche nicht allein innerhalb der Kategorie der Quantität behandeln, sondern die Kategorie der Qualität ist eine weitere konstituierende Komponente des Unendlichen und damit der Differential- und Integralrechnung. Konsequenterweise rücken nun zwei Beziehungen ins Zentrum des Interesses: einmal die Beziehung von philosophischer und mathematischer Argumentation, sodann die Beziehung von Quantität und Qualität. Für diese zweite Beziehung, also die von Quantität und Qualität, hat Hegel eine eigenständige Theorie zumindest im Rohbau entworfen, nämlich die Wissenschaft des Maßes. Innerhalb dieser Theorie könnte dann auch die Mathematik eine philosophische Heimat finden: „Die wahrhaft philosophische Wissenschaft der Mathematik als Größenlehre würde die Wissenschaft der Maße sein ... sie würde aber wohl wegen der äußerlichen Natur der Größe die allerschwerste Wissenschaft sein. ${ }^{533}$ Eine Ausarbeitung

\footnotetext{
${ }^{48}$ HW IX, S.46/47

${ }^{49}$ HW IX, S.52

${ }^{50}$ Ebd.

${ }^{51}$ HW V, S.296

${ }^{52}$ HW IX, S.54

${ }^{53}$ Ebd.
} 
dieser allerschwersten Wissenschaft hat Hegel leider nicht geleistet, ja nicht einmal begonnen.

\section{Mathematik als Exempel der Philosophie: Schopenhauer}

Kaum ein Philosoph ist wegen seiner Äußerungen zur Mathematik von den Mathematikern so heftig attackiert worden wie Schopenhauer. So rügt Alfred Pringsheim in seiner Festrede anläßlich einer öffentlichen Sitzung der Königlich Bayerischen Akademie der Wissenschaften ,das unglaublich niedrige Niveau, auf welches Schopenhauer bei seinem Feldzuge gegen die Mathematik herabsteigt ${ }^{64}$. Nun lesen sich Schopenhauers Anmerkungen zur Mathematik in der Tat wie Prolegomena zu einer jeden künftigen Mathematik, die nicht als beweisende Wissenschaft wird auftreten müssen. Aber man darf eben diese Passagen nicht isoliert, sondern nur im Kontext jener philosophischen Umgebung lesen, in welche sie von Schopenhauer mit Bedacht hineingerückt worden sind. Allein eine extrem gedeutete, fast schon verbogene Mathematik nämlich kann der Rolle gerecht werden, die Schopenhauer ihr als Exempel zur Verdeutlichung und Klärung seiner philosophischen »Grundgedanken« zugedacht hat. An drei zentralen Stellen stellt Schopenhauer die Mathematik in den Dienst seiner philosophischen Argumentation:

- in seiner Theorie der Vorstellung

- in seiner Lehre vom zureichenden Grund

- in seiner Wissenschaftslehre.

Hier wird nur die Dissertation Über die vierfache Wurzel des Satzes vom zureichenden Grunde betrachtet.

Schopenhauers Lehre vom zureichenden Grund kann aufgefaßt werden als die Theorie einer Relation auf der Klasse aller Objekte. Bei dieser Theorie stehen gewisse Paare von Objekten in der Grund-Folge-Relation: ,... alle Objekte stehn wesentlich als solche in einer gesetzmäßigen und ihrer Form nach a priori bestimmbaren Verbindung untereinander. - Diese Verbindung ist also diejenige Relation welche der Saz vom Grund allgemein genommen ausdrückt. ${ }^{65}$

Die Objekte werden in vier Klassen eingeteilt und dann auf ihre Relation hin untersucht. Für uns sind nur die zweite und dritte Klasse von Bedeutung: einmal die Klasse der abstrakten, begrifflichen Objekte - sodann die Klasse von Objekten reiner Anschauung in Raum und Zeit. In der Klasse der abstrakten Objekte besteht eine nicht-symmetrische Grund-Folge-Relation; hier sind die Rollen von Grund und Folge nicht austauschbar. In der Klasse der Objekte reiner Anschauung gibt es eine symmetrische Grund-FolgeRelation; hier sind Grund und Folge austauschbare Prädikate. Für die nicht-symmetrische Relation ist der Grund des Erkennens zuständig, für die symmetrische Relation der Grund des Seins.

Die sorgsame Trennung in die beiden geschilderten Objektklassen kann als zentrales philosophisches Anliegen der Dissertation aufgefaßt werden. Deshalb bemüht Schopenhauer ein Exempel zur Illustration, und zwar die Geometrie - genauer: seine eigene Deutung von Geometrie. Dabei vertritt er die These, daß Euklids Auffassung der Geo-

\footnotetext{
${ }^{54}$ Jahresbericht der DMV Bd.13 (1904), S.369

${ }^{55}$ Schopenhauer: Philosophische Vorlesungen I, S.444
} 
metrie - die ja noch im 19. Jahrhundert akzeptiert wurde - der Sache nach falsch ist. Euklids Geometrie in axiomatisch-deduktiver Gestalt unterliegt seiner Meinung nach den Prinzipien des Erkennens mit einer nicht-symmetrischen Grund-Folge-Relation. Für Schopenhauer gehört die Geometrie jedoch in den Wirkungsbereich des Seins. Diese Ansicht demonstriert er unter anderem am ersten Kongruenzsatz und am Lehrsatz des Pythagoras. Dabei formuliert er keineswegs zimperlich: „Jenen Eukleidischen stelzbeinigen, ja hinterlistigen Beweis hier zu wiederholen wäre zu weitläufig... “. ${ }^{56}$ Seine von ihm selbst aufgestellte Forderung nach intuitiv-anschaulicher Einsicht in den „Seynsgrund“ löst er zwar nur für den Spezialfall eines gleichschenkligen rechtwinkligen Dreiecks ein, doch fügt er sogleich hinzu: „Auch bei ungleichen Katheten müßte sich die Sache anschaulich machen lassen“. Ganz allgemein müsse in der Geometrie der Seinsgrund ,überall aufzuweisen und auch die verwickeltesten Lehrsätze auf so einfache Anschauung ulrückzuführen seyn“. 57

Man braucht sich Schopenhauers Auffassung von Geometrie keineswegs anzuschließen, um gleichwohl aus diesem Exempel zu lernen, worin Schopenhauer den fundamentalen Unterschied zwischen dem Grund des Erkennens und dem Grund des Seins sieht.

\section{Mathematik als Klient der Philosophie: Heidegger}

Schon in seinem Übersichtsbeitrag Neuere Forschungen über Logik, den er als zweiundzwanzigjähriger Student ein Jahr vor seiner Promotion in der Literarischen Rundschau für das Katholische Deutschland veröffentlicht, signalisiert Heidegger ein starkes Interesse für ,die philosophischen Probleme der Mathematik und reinen Naturwissenschaft, die seit Descartes nicht mehr zur Ruhe gekommen und durch die ungeahnten Fortschritte der Mathematik verwickelter geworden sind. ${ }^{58}$

Das hier vom jungen Heidegger artikulierte Interesse an philosophischen Problemen der Mathematik hat bei ihm das ganze Leben lang vorgehalten. Deshalb überrascht es nicht, daß man beim Studium seines Werks immer wieder auf mathematische Spuren stößt - und zwar auch an Stellen, wo man dies nicht unbedingt erwartet, etwa in seiner Nietzsche-Vorlesung Über die ewige Wiederkehr des Gleichen. Mitunter schlägt er recht schrille Töne an; so heißt es in seiner Ontologie-Vorlesung, die er 1923 in Freiburg gehalten hat: ,Mathematik ist die am wenigsten strenge Wissenschaft, denn der Zugang ist hier der allerleichteste. Geisteswissenschaft setzt viel mehr Erkenntnis voraus, als sie ein Mathematiker je erreichen kann. ${ }^{659}$

Heideggers Philosophieren läßt sich in drei längere Phasen einteilen. In der ersten Phase steht die Ausarbeitung einer Fundamentalontologie im Zentrum seiner Bemühungen; hier befreit sich Heidegger von seinen philosophischen Lehrern Husserl und Rikkert. Dann folgt, etwa vom Ende der zwanziger bis in die vierziger Jahre, eine intensive Auseinandersetzung mit der Metaphysik. Die Spätphilosophie Heideggers schließlich wird wesentlich durch eine philosophische Analyse und Kritik der Technik bestimmt. In allen drei Phasen finden sich mathematische Spuren in Heideggers Werk - und stets sind

\footnotetext{
${ }^{56}$ Ebd. S.455

${ }^{57}$ Ebd. S.453

${ }^{58}$ Heidegger: Gesamtausgabe Bd.1, S.43

${ }^{59}$ Ebd., Bd.63, S.72
} 
diese Spuren so signifikant durch die jeweilige Phase geprägt, daß man den Äußerungen zur Mathematik auch ohne Kenntnis ihrer Plazierung ansehen kann, aus welcher Phase sie stammen. Durchgängig verfolgen läßt sich jedoch eine ganz bestimmte methodische Vorgehensweise, indem nämlich aus der jeweiligen philosophischen Position heraus Aussagen über Struktur oder Ursprung mathematischer Erkenntnis abgeleitet werden. Stets erscheint die Mathematik als Klient der Philosophie. Ein recht typisches Exempel ist der Satz „Die mathematische Erkenntnis gilt als diejenige Erfassungsart von Seiendem, die der sicheren Habe des Seins des in ihr erfaßten Seienden jederzeit gewiß sein kann ${ }^{660}$.

In seiner zweiten, durch Metaphysik geprägten Phase beschäftigt sich Heidegger besonders intensiv mit Mathematik. Im Rahmen dieses Vortrags sind jene Überlegungen nicht zuletzt deshalb von Interesse, weil dabei erneut auf die Entdeckung der Möglichkeit von Wissenschaft am Beispiel der griechischen Mathematik eingegangen wird. Als zentralen neuen Begriff führt Heidegger das Mathematische ein: „Die entscheidende Frage lautet: Was heißt hier »Mathematik « und »mathematisch«? Es scheint, daß wir die Antwort auf diese Frage nur aus der Mathematik selbst schöpfen können. Das ist ein Irrtum; denn die Mathematik ist selbst nur eine bestimmte Ausformung des Mathematischen. ${ }^{\text {(61 }}$ Bei diesem Ansatz muß also vorab - unabhängig von Mathematik - das Mathematische bestimmt und daran anschließend Mathematik als eine ,bestimmte Ausformung des Mathematischen“ gedeutet werden. Deshalb fragt Heidegger folgerichtig: „Wie ist es mit dem »Mathematischen«, wenn es nicht aus der Mathematik erklärt werden kann? Bei solchen Fragen tun wir gut, uns an das Wort zu halten ... Das »Mathematische« kommt der Wortprägung nach vom griechischen $\tau \alpha \mu \alpha \vartheta \eta \mu \alpha \tau \alpha \ldots . .{ }^{{ }^{62}}$ Das Mathematische versucht Heidegger also durch einen Rückgang auf die klassische Antike in den Griff zu bekommen, indem er dem damaligen Paradigmenwechsel im Umgang mit Wissen eine neuartige Deutung gibt.

Die Griechen haben, wie hinlänglich bekannt, völlig neue Formen des Wissens auf den Weg gebracht und diese verschiedenen Arten des Wissens dann begrifflich zu fassen versucht: $\mu \alpha \vartheta \eta \sigma i \varsigma$ heißt bei ihnen das Lernen, $\mu \alpha \vartheta \eta \varepsilon \mu \alpha \tau \alpha$ das Lernbare. Dies ist $\mathrm{zl}-$ nächst nicht an bestimmte Inhalte - etwa Geometrie oder Arithmetik - gebunden. Heidegger unterscheidet nun ein Üben von bekanntem Wissen und ein Kennenlernen von neuem Wissen - Routine im Umgang mit verfügbarem Wissen und Konstitution von neuem Wissen. Das Mathematische steht bei ihm für eine ganz bestimmte Art und Weise der Konstitution von neuem Wissen: „Die $\mu \alpha \vartheta \eta \varepsilon \mu \alpha \tau \alpha$, das sind die Dinge, sofern wir sie in die Kenntnis nehmen, als was wir sie eigentlich im voraus schon kennen ... Wir nehmen, was wir irgendwie schon selbst haben. Es handelt sich um solches Lernbare, was als Mathematisches begriffen werden muß.“63 Hier hat also das wahr-nehmende Subjekt absolute Priorität gegenüber dem wahr-nehmbaren Objekt. Diese Aktivität auf der Subjektseite unterstreicht Heidegger durch die Aussage: „Im Wesen des Mathematischen ... liegt ein eigentümlicher Wille zur Neugestaltung und Selbstbegründung der Wissens-

\footnotetext{
${ }^{60}$ Heidegger: Sein und Zeit, S.95

${ }^{61}$ Heidegger: Die Frage nach dem Ding, S.52

${ }^{62}$ Ebd., S.53

${ }^{63}$ Ebd., S.56/58
} 
form als solcher. “64 Das hier in knapper Skizze geschilderte Mathematische ist selbstverständlich Ursprung und Anlaß für Mathematik, jedoch - und darin liegt die Pointe der Heideggerschen Argumentation - keineswegs nur für Mathematik, denn für ihn ,... sind die neuzeitlichen Naturwissenschaften und die neuzeitliche Mathematik und die neuzeitliche Metaphysik aus derselben Wurzel des Mathematischen entsprungen. “65 Damit erfährt nun auch die Mathematik eine völlig neue Ortsbestimmung innerhalb eines umfassenderen Rahmens. Als Philosoph ist Heidegger mehr an der Philosophie als an der Mathematik interessiert; deshalb fügt er noch programmatisch hinzu: „Weil unter diesen dreien die Metaphysik am weitesten ausgreift ... und weil sie zugleich am tiefsten greift ... muß gerade die Metaphysik ihren mathematischen Grund und Boden bis auf den Felsgrund ausschlachten." Uns interessiert natürlich vorrangig, wie denn nun die neuzeitliche Mathematik durch das Mathematische initiiert wird. Dazu äußert sich Heidegger leider nur recht knapp und pauschal: „Daß eine Mathematik, und zwar eine solche besonderen Schlages, ins Spiel kommen konnte und mußte, ist die Folge des mathematischen Entwurfs. Die Begründung der analytischen Geometrie durch Descartes, die Begründung der Fluxionsrechnung durch Newton, die gleichzeitige Begründung der Differentialrechnung durch Leibniz, all dies Neue, dieses Mathematische im engeren Sinne, wurde erst möglich und vor allem notwendig auf dem Grunde des mathematischen Grundzugs des Denkens überhaupt. ${ }^{666}$ Mit diesen globalen Hinweisen zur Mathematik hat es bei Heidegger sein Bewenden, an dieser Stelle endet seine Markierung bzw. Richtungsangabe für den Weg vom Mathematischen zur Mathematik. Mir ist kein Versuch bekannt, diesen Weg wirklich zu gehen und darüber zu berichten. Ich meine aber, daß es einen Versuch wert wäre.

Als Einstieg in einen simultanen Rückblick auf alle acht angesprochenen Philosophen erinnere ich an Schellings Studienanleitung: ,Will man einen Philosophen ehren, so muß man ihn da auffassen, wo er noch nicht zu den Folgen fortgegangen ist, in seinem Grundgedanken ... Der wahre Gedanke eines Philosophen ist eben sein Grundgedanke, der von dem er ausgeht." Im Anschluß an die geschilderten mathematischen Spuren halte ich die These für gerechtfertigt, daß in allen acht Fällen der philosophische Grundgedanke eine mathematische Komponente hat. Dies steht nicht im Widerspruch zu Kants Hinweis in seinem opus postumum, daß es weder mathematische Anfangsgründe der Philosophie noch philosophische Anfangsgründe der Mathematik gibt. Nein, von mathematischen Anfangsgründen ist nicht die Rede, doch eine Orientierungsfunktion der Mathematik für die jeweilige philosophische Konzeption - für den philosophischen Grundgedanken kann meines Erachtens nicht geleugnet werden. Intention oder Zweck dieser Orientierung variieren von Philosoph zu Philosoph, in der Art und Weise des Zugriffs auf Mathematik ist jedoch eine methodische Gemeinsamkeit nicht zu übersehen: Es wird stets die Mathematik einer eigenständigen - mitunter auch eigenwilligen - Deutung unterzogen, damit die so präparierte Mathematik dem Grundgedanken des Philosophen Überzeugungskraft verleiht und die neue philosophische Konzeption verständlich werden läßt. In

\footnotetext{
${ }^{64}$ Ebd., S.75

${ }^{65}$ Ebd., S.75

${ }^{66}$ Ebd., S.72
} 
den meisten Fällen wird dabei tradierte Mathematik auf eine völlig neue Art und Weise interpretiert; hier gilt es dann, Anlaß und Intention dieser neuen Interpretation zu bestimmen.

Bei Hegel beobachten wir zusätzlich eine engagierte Auseinandersetzung mit der stürmischen Entwicklung der Analysis zu seiner Zeit. Die erste Auflage von Hegels Wissenschaft der Logik erschien 1812, eine wesentlich überarbeitete zweite Auflage kam 1832 heraus. In der Zwischenzeit war Cauchys Cours d'analyse veröffentlicht und Cauchys neue Präsentation der Analysis hat Hegel bei der Neubearbeitung seiner Wissenschaft der Logik stark beeinflußt. Die Lehre vom Maß, sie handelt von der Relation zwischen Quantität und Qualität, nimmt in der ersten Auflage nur wenig Raum ein, wird in der zweiten Auflage jedoch wesentlich ausführlicher entfaltet. Insbesondere wird die Abhängigkeit quantitativer und qualitativer Änderungen im Detail analysiert. Und hier ist der Einfluß des von Cauchy neu geprägten und präzisierten Stetigkeitsbegriffs unübersehbar. Eine Änderung bloß der Quantität bei Invarianz der Qualität schildert Hegel so: „Die Veränderung ist nach dieser Seite eine allmähliche. ${ }^{607}$ Diese Art der Änderung entspricht der Stetigkeit. Die Unstetigkeit findet ihr philosophisches Korrelat in der Weise, daß eine quantitative Änderung eine qualitative Änderung impliziert: „Nach der qualitativen Seite wird daher das bloß quantitative Fortgehen der Allmählichkeit ... absolut abgebrochen; indem die neu eintretende Qualität nach ihrer bloß quantitativen Beziehung eine gegen die verschwindende unbestimmt andere, eine gleichgültige ist, ist der Übergang ein Sprung. ‘68 Allmähliche Veränderungen der Quantität können also die ihr verbundene Qualität entweder invariant lassen oder aber ändern; im Fall einer Änderung erfolgt der Übergang nach Hegel als Sprung. Offensichtlich haben mathematische Denkformen sowohl die Konzeption als auch die Terminologie dieser philosophischen Lehre vom Maß geprägt.

\section{Literatur}

Gaiser, K.: Platons Zusammenschau der mathematischen Wissenschaften. Antike und Abendland Bd.32 (1986), S.89-124

Hegel, G. W. F.: Theorie - Werkausgabe. Frankfurt/M.: Suhrkamp 1970

Heidegger, Martin: Gesamtausgabe. Frankfurt/M.: Klostermann $1975 f f$.

Heidegger, Martin: Sein und Zeit. Tübingen: Niemeyer 1962

Heidegger, M.: Die Frage nach dem Ding. Tübingen: Niemeyer 1962

Hilbert, D.: Gesammelte Abhandlungen. Berlin - Heidelberg - New York: Springer 1979

Kant, I.: Werke, hrsg. von W. Weischedel. Darmstadt: Wissenschaftliche Buchgesellschaft 1983

Mittelstraß, J.: Die geometrischen Wurzeln der platonischen Ideenlehre. Gymnasium Bd.92 (1985), S.399-418

Nikolaus von Kues: Dreiergespräch über das Können - Ist. Hamburg: Meiner 1973

\footnotetext{
${ }^{67}$ HW 5, S.295.

${ }^{68}$ HW 5, S.438
} 
Nikolaus von Kues: Die belehrte Unwissenheit II. Hamburg: Meiner 1977

Nikolaus von Kues: Über den Beryll. Hamburg: Meiner 1977

Nikolaus von Kues: Die belehrte Unwissenheit I. Hamburg: Meiner 1979

Nikolaus von Kues: Die mathematischen Schriften. Hamburg: Meiner 1980

Nikolaus von Kues: Mutmaßungen. Hamburg: Meiner 1988

Novalis: Schriften. Die Werke Friedrich von Hardenbergs, hrsg. von R. Samuel in Zusammenarbeit mit H.-J. Mähl und G. Schulz. Darmstadt: Wissenschaftliche Buchgesellschaft 1977ff.

Patzig, G.: Tatsachen, Normen, Sätze. Stuttgart: Reclam jun. 1980

Platon: Sämtliche Werke. Hamburg: Rowohlt 1959ff.

Radbruch, K.: Anschauung und Beweis in der Mathematik. Skeptische Anmerkungen zum Optimisten Schopenhauer. Schopenhauer-Jahrbuch Bd.69 (1988), S.119-.126

Radbruch, K.: Mathematik in den Geisteswissenschaften. Göttingen: Vandenhoeck\&Ruprecht 1989

Radbruch, K.: Ein Nonstandard-Blick auf die Mathematik bei Hegel. Hegel-Jahrbuch 1989, S.249255

Radbruch, K.: Die Mathematik im Werk von Heidegger und Jaspers - ein aufschlußreicher Vergleich. Jahrbuch der Österreichischen Karl-Jaspers-Gesellschaft, Jahrgang 3/4 (1990/91), S.191203

Radbruch, K.: Mathematik à la Philosophie - Philosophie à la Mathematik: Ein historischer Überblick. Mathematische Semesterberichte Bd.38 (1991), S.18-57

Radbruch, K.: Philosophische Spuren in Geschichte und Didaktik der Mathematik. Mathematische Semesterberichte Bd.40 (1993), S.1-27

Radbruch, K.: Was kann die heutige Mathematik von Schelling lernen? Selbstorganisation. Jahrbuch für Komplexität in den Natur- Sozial- und Geisteswissenschaften Bd.5 (1994), S.55-72

Radruch, K.: Das Interesse der Philosophen an mathematischer Erkenntnis. DMV-Mitteilungen 3/1997, S.45-46

Radbruch, K.: Mathematische Spuren in der Literatur. Darmstadt: Wissenschaftliche Buchgesellschaft 1997

Schelling, F.W.: Sämtliche Werke, hrsg. von K.F.A. Schelling. Stuttgart: Cotta 1856-1861

Schlegel, Fr.: Kritische Ausgabe, hrsg. von Ernst Behler. München - Paderborn - Wien - Zürich: Schöning - Thomas 1958ff

Schopenhauer, A.: Theorie des gesamten Vorstellens, Denkens und Erkennens. Aus dem handschriftlichen Nachlaß hrsg. von V. Spierling. München - Zürich: Piper 1986

v.Weizsäcker, C.F.: Zeit und Wissen. München: Hanser 1992

Wolff, Ch.: Ausführliche Nachricht von seinen eigenen Schriften, die er in deutscher Sprache herausgegeben. Nachdruck Hildesheim: Olms 1973 